\title{
Aporte de la economía del transporte al ordenamiento territorial
}

María Emilia García Schilardi*

\section{RESUMEN}

El objetivo de este trabajo es evaluar el aporte teórico conceptual de la economía del transporte al ordenamiento territorial a través de un análisis comparado del estado actual de cada uno de estos enfoques en relación con sus principales conceptos y metodologías. Como resultado se detecta que existen diversos puntos de vinculación entre estos enfoques. Principalmente, se observa que la economía del transporte es la disciplina que aporta los conceptos, metodologías y marco teórico para conocer el comportamiento del mercado de este servicio y que el ordenamiento territorial, como política pública transversal, integra estas herramientas y las complementa con otras provenientes de diversas disciplinas.

Palabras clave: economía, transporte, territorio, movilidad.

Clasificación JEL: R41 y A12.

Consejo Nacional de Investigaciones Científicas y Técnicas, Instituto de Cartografía, Investigaciones y Formación para el Ordenamiento Territorial (CIFOT), Universidad Nacional de Cuyo, Mendoza, Argentina. Correo electrónico: maria-emilia-garcia@hotmail.com. 


\section{ABSTRACT}

\section{Contribution of the transport economy to the territorial planning}

The objective of this paper is to evaluate the conceptual and theoretical contribution of the transport economy to territorial planning through a comparative analysis of the current status of each of these approaches in relation to its main concepts and methodologies. As a result, it is detected that there are several points of connection. Mainly, it is observed that the transport economy is the discipline that provides the concepts, methodologies and theoretical framework to learn the behavior of this service's market and that the territorial planning, as transversal public policy, integrates these tools and complements them with others from diverse disciplines

Keywords: economy, transport, territory, mobility.

JEL Classification: R41 y A12.

\section{INTRODUCCIÓN}

El territorio, el transporte y la economía se entrelazan e influyen recíprocamente. El territorio se configura como un sistema, ya no una porción del espacio estático y fijo, sino un espacio geográfico dinámico, flexible en donde se movilizan y actúan personas y objetos. Este sistema está constituido, a la vez, por subsistemas entre los cuales se encuentra el transporte operando como soporte de su estructura y sus flujos. El transporte se compone de diversas dimensiones, siendo la económica una de ellas, y analizando el comportamiento del mercado de cada modo que impacta directamente en el modelo de movilidad que se desarrolla en un territorio.

Estos elementos y sus relaciones han sido abordados a lo largo del tiempo desde diversas perspectivas, en general resultando en análisis parciales, sectoriales con visiones incompletas de la realidad compleja. Ante esto, surge como enfoque innovador el ordenamiento territorial que entiende al territorio desde su naturaleza multiescalar, multidimensional y por tanto sistémica, proponiendo un bordaje holístico del mismo. Éste emerge como una política transversal e integradora de aquellas políticas de carácter sectorial. El estudio de la economía del transporte, asimismo, está sufriendo una transformación en su dimensión analítica y práctica en tanto se ha pasado de una concepción tradicional enfocada en la eficiencia de infraestructuras y servicios hacia una 
más amplia que incluye los deseos y necesidades de desplazamiento de las personas.

El objetivo de este trabajo es, entonces, evaluar el aporte teórico conceptual de la economía del transporte al ordenamiento territorial. Para ello, se realiza un análisis del estado actual de cada uno de estos enfoques en relación a sus principales conceptos y metodologías y se emplea la técnica de la comparación, contrastando las siguientes dimensiones: definición, objeto de estudio, modo de intervención, concepción del territorio, concepción del Estado, subsistemas que potencia, abordaje del transporte colectivo, recursos productivos empleados, actores intervinientes y objetivos de intervención. No obstante se busca ir más allá detectando aportes o vínculos adicionales entre estos enfoques, por lo que se analizan conceptos propios de la economía del transporte desde la perspectiva del ordenamiento territorial.

Como resultado se detecta que existen diversos puntos de vinculación entre estos enfoques. Principalmente, se observa que la economía del transporte es la disciplina que aporta los conceptos, metodologías y marco teórico para conocer el comportamiento de los componentes vinculados al sistema de transporte y que el ordenamiento territorial como política pública transversal hace uso de estas herramientas y las complementa con otras provenientes de diversas disciplinas.

El desarrollo del trabajo comienza con la relación existente entre los objetos de estudio de ambos enfoques, el territorio y el transporte. En un apartado posterior se hace una caracterización del enfoque de ordenamiento territorial y luego un detalle de los orígenes y principales exponentes de la economía del transporte. A posteriori, se realiza el análisis comparativo de las perspectivas mencionadas y se amplía identificando los conceptos de la economía del transporte que se vinculan con el ordenamiento territorial. Finalmente, se realizan unas reflexiones finales que sintetizan lo analizado en el trabajo.

\section{EL TERRITORIO Y EL TRANSPORTE}

En el análisis del aporte de la economía del transporte al ordenamiento territorial, resulta de especial interés la caracterización y vínculo entre los objetos de estudio de ambos enfoques.

Como primer paso cabe la pregunta ¿qué es el territorio? Si bien existen infinidad de caracterizaciones, como primera aproximación 
puede definirse como un espacio geográfico delimitado por pautas institucionales y legales y definido por el sentido de pertenencia de la comunidad que lo habita. En él se dan procesos naturales, históricos, culturales y económicos que llevan a conformar una estructura productiva, socioeconómica y ambiental que da identidad a un lugar. En el territorio se da la relación permanente entre los procesos sociales, económicos y ambientales que le dan identidad al mismo.

Es decir, se deja de lado la concepción estática y mercantilista del mismo y se entiende como un proceso, un espacio geográfico dinámico, flexible, en donde se movilizan y actúan personas y objetos.

Lefebvre (1978; citado en Raffestin, 1980: 50) entendía el territorio como

la producción de un espacio físico, delimitado, modificado, transformado por las redes, circuitos y los flujos correspondientes que se instalan como rutas, canales, vías férreas, circuitos comerciales y bancarios, autopistas. El territorio no es ya un objeto en el sentido del espacio, es un proceso en perpetua transformación según las escalas temporales particulares.

El territorio, bajo estas consideraciones, resulta ser un bien común en tanto que es un complejo sistema de componentes y relaciones entre el medio físico y la sociedad que lo habita. Por ello, no puede excluirse a ninguna persona de la actuación en el mismo y el hecho de que un habitante sea partícipe no impide que otro también lo sea. En palabras de Romero y Vázquez (2005: 91):

De allí que el territorio deba ser conceptualizado esencialmente como un bien común, lo que constituye la razón por la que los estados asumen la formulación de políticas públicas para su administración y mantienen aún grandes extensiones bajo el dominio público o bien son objeto de regulaciones especiales dado su carácter y naturaleza como bien común.

Por su parte Santos (1996) entiende al territorio como un entramado indivisible, complejo y contradictorio, de sistemas de objetos y sistemas de acciones. Se observa que este autor coincide con la idea de territorio como sistema.

Los sistemas de objetos y los sistemas de acciones interactúan, por un lado, los objetos condicionan la forma como se llevan a cabo las 
acciones y, por otro lado, los sistemas de acciones conducen a la creación de objetos nuevos o se realizan sobre objetos preexistentes (Santos, 2000: 32).

Uno de los componentes u objetos estructurantes del territorio es la movilidad. Un territorio tiene componentes morfológicos, estructurales y funcionales y la movilidad forma parte del funcionamiento o comportamiento de los mismos, da cuenta de su dinámica, a la vez que forma parte de su estructura.

La movilidad, asimismo, contiene como un elemento constituyente al transporte. En palabras de Gutiérrez (2009: 4): “en general, el transporte es entendido como el componente material de la movilidad, ligado a los medios técnicos de desplazamiento. Y la movilidad como algo que se expresa en el transporte". La movilidad resulta, entonces, un concepto más amplio que el de transporte. En términos de un silogismo hipotético si uno de los componentes del territorio es la movilidad, si el transporte es un objeto de ésta, entonces, el transporte es un elemento constitutivo del territorio.

Siguiendo en la argumentación respecto de la movilidad y el transporte, Herce (2009: 51-52) afirma:

[...] "transporte" y "movilidad" no son términos sinónimos [...]. El primer concepto implica dispendio de energía y, por tanto, creación de infraestructuras especializadas para intentar abaratar el costo del viaje (o de empresas especializadas en producir esas infraestructuras); y, por el contrario, el segundo supone atención a las formas más autónomas de desplazamiento, oferta de alternativas posibles, gestión del gasto energético y del espacio desde una perspectiva más amplia que la de la recuperación del coste invertido o la de la adaptación de la capacidad de la infraestructura a la demanda de viajes en un determinado medio de transporte.

La movilidad se entiende como el acceso de los ciudadanos a los servicios urbanos como salud, educación, trabajo, ocio mediante diversos modos de transporte: a pie, bicicleta, vehículo particular motorizado, transporte público. En general, los estudios sobre transporte se basan en el viaje realizado, que muestra sólo parcialmente la movilidad de una sociedad.

En la consideración particular del territorio urbano, "los conceptos de movilidad y transporte sostenibles se sitúan, por supuesto, en 
el núcleo de la investigación sobre la sostenibilidad urbana, como elementos fundamentales que son de la estructura y funcionamiento de las ciudades" (Salado García, et al., 2002: 1778).

Como ya se mencionó, el objeto de estudio del ordenamiento territorial es el territorio; mientras que el objeto de estudio de la economía de transporte es, precisamente, el transporte, el comportamiento de su mercado, de sus actores, de su oferta y demanda. Estos dos objetos de estudio se vinculan a través de una relación mutua. El mercado de transporte, a través de la dinámica de las fuerzas de oferta y demanda que lo componen, genera un impacto en el territorio en donde se desarrolla. Impacta en la distribución de usos del suelo, por lo que el territorio urbano se establece como una dimensión intrínseca de los sistemas de transporte. Asimismo, la distribución de la población y los usos del suelo tienen impacto en el mercado de transporte (sea este positivo o negativo) por lo que influyen en las acciones que el Estado define para regular este mercado. La localización de las actividades urbanas, sobre el territorio, condicionan el sentido y la cantidad de los desplazamientos.

Según Miralles Guasch (2002), el transporte y el territorio se entrecruzan e influyen mutuamente, estableciendo una relación de reciprocidad de tal modo que al mismo tiempo que la distribución de los usos del suelo configura determinados patrones de movilidad, influyendo en los diversos modos de transporte, la oferta de transporte presente en un territorio puede condicionar los patrones de localización de las actividades urbanas.

De este modo, los procesos de transformación que ocurren en el territorio urbano están influenciados por múltiples factores no aislados individualmente, entre ellos el sistema de transporte urbano, sumándose, superponiéndose y generando interrelaciones y sinergias entre sí (García, 2007: 123).

El estudio del vínculo transversal e integral de estas dos variables tiene pocos precedentes en América Latina. Prácticamente no existe un análisis sobre el tema que incorpore una visión integrada y articulada de dichos conceptos (Montezuma, 1999). 


\section{2. ¿ORDENAMIENTO TERRITORIAL?}

La primera expresión de ordenamiento territorial aparece en la Carta Europea de Ordenación del Territorio (1983: 2) que lo define como:

La expresión espacial de la política económica, social, cultural y ecológica de toda sociedad, con multitud de objetivos, entre ellos el desarrollo socio económico y equilibrado de las regiones, la mejora de la calidad de vida, la gestión responsable de los recursos naturales, la protección del medio ambiente y, por último, la utilización racional del territorio. Es a la vez una disciplina científica, una técnica administrativa y una política concebida como un enfoque interdisciplinario y global, cuyo objetivo es un desarrollo equilibrado de las regiones y la organización física del espacio según un concepto rector.

El ordenamiento territorial es una expresión polisémica por lo que resulta difícil definirla de manera taxativa. Existe coincidencia entre los autores de que el concepto gira en torno a tres elementos: las actividades humanas, el espacio geográfico en el que se desarrollan y el sistema que ambos constituyen. En este sentido, ordenar un territorio implica identificar, distribuir, organizar y regular las actividades humanas en un territorio de acuerdo con ciertos criterios y prioridades, se podría hablar de ordenación de las actividades humanas en un espacio geográfico capaz de acogerlas (Gómez et al., 2014 y Gómez, 2002). Asimismo, existe acuerdo en el objeto del mismo:

La idea de regular u organizar el uso, ocupación y transformación del territorio en pro de su aprovechamiento óptimo. Este aprovechamiento se asocia generalmente con el uso racional y sustentable de los recursos naturales (planificación física ambiental), en estrecha correspondencia con patrones adecuados de distribución de asentamientos y de actividades económicas (Massiris, 2005: 15).

Este modo de abordar el territorio emplea las metodologías propias de las disciplinas desde la que se aborda el territorio, y a través de la interdisciplina estas metodologías resultan coherentes y complementarias. Esto no implica, en consecuencia, la existencia de una metodología propia del ordenamiento del territorio, por lo que no resultaría 
acertado postularlo como una disciplina científica, como se promueve en la Carta Europea de Ordenación del Territorio (1983). Al respecto Almeida García (1999: 55) afirma:

La ordenación del territorio como tarea interdisciplinar no tiene una única ciencia que se arrogue el privilegio de poder estudiarla en exclusividad. El gran número de elementos de los que se compone el territorio imposibilita la comprensión de la realidad a partir de una única ciencia. Si la realidad territorial es compleja y diversa no se la puede abordar desde la parcialidad.

Existe acuerdo, por otro lado, en que el ordenamiento territorial se constituye como una técnica administrativa que requiere una acción voluntaria y coordinada de intervención en las distintas dimensiones del modelo de organización territorial. Para ello se hace necesario, en palabras de Gudiño (2002: 3):

[...] la redefinición de la estructura administrativa actual a partir de la propuesta de una estructura flexible organizacional que permita mejorar la capacidad de gestión a partir de la incorporación de tecnología informacional, una planificación administrativa-contable, un O.T. adaptado a las demandas actuales con una adecuada capacitación del recurso humano interviniente en el proceso.

Esto requiere de nuevas modalidades de gestión por parte del Estado para hacer frente a los problemas territoriales complejos.

Finalmente, el ordenamiento territorial se conforma como una política pública aunque con una concepción más amplia que las políticas sectoriales ya que es transversal a ellas, las comprende e integra. Requiere, entonces, una visión interjurisdiccional y un proceso constante, interactivo y participativo de todos los actores, tanto públicos como privados. Necesita, en definitiva, constituirse como una política de Estado. Massiris (1997: 11) destaca:

Las experiencias y conceptualizaciones sobre ordenamiento territorial en el mundo permiten colegir que se trata de una política de Estado y un proceso planificado de naturaleza política, técnica y administrativa, cuyo objeto central es el de organizar, armonizar y 
administrar la ocupación y uso del espacio, de modo que éstos contribuyan al desarrollo humano ecológicamente sostenible, espacialmente armónico y socialmente justo. Lo anterior pone en evidencia que en el ordenamiento territorial confluyen las políticas ambientales, las políticas de desarrollo regional, espacial o territorial y las políticas de desarrollo social y cultural, cuya naturaleza es determinada por el modelo de desarrollo económico dominante en cada país.

Asimismo, Gudiño (2015) plantea la necesidad de que el ordenamiento del territorio sea una política de Estado, entendiendo que éstas resultan

Estables y continuas. No dependen de los cambiantes humores políticos de los gobiernos, ni de las coyunturas electorales, sino que se apoyan en saberes técnicos y en consensos. Hoy, las políticas de Estado aparecen en muchas propuestas como un programa redactado por expertos y rubricado por políticos y en las antípodas del decisionismo. Combinan la ilusión tecnocrática con una dosis de otra ilusión: el consenso (Romero, 2010, citado por Gudiño 2015: $17-18)$.

Esta técnica administrativa y política de Estado tiene como finalidad la mejora en la calidad de vida de las personas (Aguilar, 1989; Méndez, 1990; Poinsont, 1991; Massiris, 1991; Gómez, 2002, citado en Massiris, 2005), y para ello busca un desarrollo uniforme de todas las dimensiones que lo configuran (social, económica y ambiental).

Todo territorio tiene un modelo de ordenación que está en directa relación con el modelo de desarrollo del mismo. El orden territorial es el resultado de acciones públicas y privadas, estén éstas más orientadas al libre funcionamiento del mercado o enmarcadas en una política de Estado y políticas públicas que promuevan un orden determinado. El ordenamiento del territorio no intenta destruir al mercado, sino conocerlo, entenderlo, preverlo y finalmente participar de él para influirlo y guiarlo para atenuar las disparidades detectadas. Gudiño (2009: 5) plantea que el ordenamiento del territorio comienza a centrar su atención en

la producción y la relación público-privado. Esto plantea una cierta complejidad del proceso: armonizar la acción del sector público con el interés de los agentes privados también ordenadores del 
territorio, así como armonizar el interés privado con el interés colectivo y conciliar los objetivos económicos con los del ordenamiento del territorio.

Como corolario, se entiende que el ordenamiento territorial reconoce las fuerzas de oferta y demanda que actúan en los mercados, en particular en este desarrollo interesan aquellas vinculadas a la movilidad y al transporte que se conocen y analizan desde los conceptos y teorías propuestos por la economía del transporte.

\section{ORÍGENES Y PRINCIPALES AUTORES DE LA ECONOMÍA DEL TRANSPORTE}

La economía del transporte se origina de la mano de las teorías de la localización de la actividad económica, en las cuales se considera como componente fundamental de la distribución a los costos de transporte. Esto se observa desde los modelos clásicos y neoclásicos (Von Thünen, 1826; Weber, 1909; Hotelling, 1929; Reilly, 1931; Christaller, 1940; Hoover, 1943; Isard, 1956; Moses, 1958) hasta los desarrollos más recientes de la Nueva geografía económica (Krugman, 1991). Estos estudios buscan determinar, bajo algunos supuestos que van modificando, la localización óptima de la actividad económica en términos de la minimización de costos de transporte. Algunas generalizaciones y extensiones más actuales sostienen que la atracción de una localización depende tanto del tamaño relativo de su mercado, como de la capacidad y calidad de su red de transporte (Combes, Mayer y Thisse, 2008; Ottaviano; 2008 y Puga, 2009).

Asimismo, en la década de los cuarenta del siglo XX, aparecen los modelos de transporte elaborados principalmente desde las lógicas de la ingeniería y la logística, pero también desde la economía en términos de que buscan satisfacer la distribución de productos al mínimo costo de transporte. El primer abordaje es el de Hitchcock (1941) quien estudia la distribución de un producto desde diversos orígenes a numerosos destinos. Luego en 1947 aparece el trabajo de Koopmans en el que se estudia la utilización óptima del sistema de transporte, profundizando en las características y comportamiento de dicho sistema. Estos modelos emplean a la programación lineal como el método de cálculo y estimación. En el año 1990, Ortúzar y Willumsen aportan una sistematización para la modelización y planificación del transporte en la 
que se consideran características y comportamientos de variables que pertenecen al mercado de transporte; principalmente se desarrolla el modelo de demanda, pero también se abordan cuestiones de oferta y de equilibrio.

Es en el año 2003, con el trabajo de DeRus, Campos y Nombela, que surge por primera vez una integración de todos los conceptos y métodos propios de la economía del transporte. Esta obra aporta una descripción pormenorizada de los temas centrales de la economía del transporte, con una perspectiva integrada de las cuestiones relevantes que facilita el abordaje de un sector tan complejo y transversal. Asimismo, en el año 2008 aparece el trabajo de Mendieta y Perdomo (2008), que realiza aportes de diferentes teorías y metodologías que estudian el sector transporte desde una perspectiva económica. Estos dos últimos abordajes se constituyen en los más completos y actualizados que abordan la economía del transporte.

El marco teórico general de la economía del trasporte responde a los postulados de la teoría microeconómica. No obstante, y siguiendo lo propuesto por De Rus, Campos y Nombela (2003), esta rama especializada de la economía opera considerando unos principios fundamentales, de 1 siguiente forma. Por otro lado, la existencia y diferenciación de dos funciones de oferta: la de servicios y la de infraestructura; cada una de las cuales requiere de un estudio particular y de una identificación especial de su demanda; resultando especialmente importante el cálculo de la inversión óptima en infraestructura por la envergadura de la misma y por el impacto en otras actividades y en el territorio. Asimismo, se detecta que el transporte es un servicio intermedio, es decir, es útil en tanto permite realizar otras actividades y por ello el tiempo de los usuarios es un recurso fundamental tanto para la función de oferta como de demanda. Los servicios de transporte tienen la particularidad de ser no almacenables y de oferta no continua, por lo que es clave la predicción adecuada de la demanda en tiempo y espacio. Adicionalmente, dado que el mercado de transporte no cumple con los requisitos de competencia perfecta es necesaria su regulación para alcanzar niveles de producción cercanos al óptimo. Por su parte, el transporte funciona como un sistema y, por ello, se producen economías de red; esto requiere ineludiblemente de un abordaje integral del mismo. A su vez, el funcionamiento del transporte genera altas externalidades negativas que se trasladan a la sociedad si no se establecen mecanismos 
de internalización. En la producción de servicios de transporte se generan tres tipos costos (de producción, del usuario y sociales); resulta clave en el comportamiento del mercado determinar quién los paga. Se entiende que el transporte es un servicio público de primera necesidad y como tal no solamente se rige por criterios de eficiencia económica sino también por criterios de equidad. Finalmente, se detecta que el transporte se constituye como un componente clave para la movilidad de las personas y los bienes por lo que se considera tanto como un elemento de la microeconomía como de la macroeconomía: de la primera dado que es esencial para el desarrollo de la vida cotidiana; de la segunda, por su importancia en la estructura productiva de las naciones.

\section{ANÁLISIS COMPARATIVO DE LOS ENFOQUES}

La economía del transporte asume los mismos conceptos y postulados de la teoría microeconómica. Particularmente, estudia el comportamiento del mercado de cada modo de transporte. A partir de esta consideración se analizan a continuación, en clave comparada, este enfoque y el de ordenamiento territorial (tabla 1).

Una diferencia fundamental de estas dos perspectivas es que el ordenamiento territorial se constituye como una política pública y como una técnica administrativa, mientras que la economía del transporte es una disciplina técnico social proveniente de la teoría microeconómica, que se basa en conceptos, metodologías y teorías específicas. El ordenamiento territorial, por el contrario, en su carácter interdisciplinar, hace uso de dichos conceptos, metodologías y teorías y busca combinarlos de manera sistémica con aquellos propuestos por otras disciplinas. Es decir, el ordenamiento territorial emplea los postulados de las diversas disciplinas y los conjuga y complementa, en la medida que no sean contradictorios, para a partir del ellos generar políticas públicas integradas y transversales.

Partiendo del vínculo estrecho entre el transporte y el territorio, se entiende que estas perspectivas resultan complementarias y se enriquecen entre sí. El ordenamiento territorial requiere del análisis de la dimensión económica del territorio y del transporte, necesita conocer el funcionamiento de los mercados para identificar el impacto en el territorio. A la vez, completa este análisis con otras dimensiones que 
permiten que la perspectiva disciplinar y sectorial se amplíe hacia una visión más integral.

Lo anterior implica que el modo de intervención que se propone desde ambos enfoques no sea el mismo. El ordenamiento territorial considera que el territorio tiene una naturaleza multiescalar, sistémica e integral (Gudiño, 2010) y por ello las políticas públicas en ordenamiento territorial adquieren una concepción más amplia que las políticas sectoriales ya que son transversales. Son propuestas con una visión interjurisdiccional y de un proceso constante, interactivo y participativo de todos los actores, tanto públicos como privados. En cambio, la economía del transporte mantiene una visión sectorial y economicista de las intervenciones, que omiten la naturaleza integral del territorio. Se detecta, entonces, que el ordenamiento territorial se esgrime como una planificación holística del territorio que contiene y emplea a los instrumentos económicos y los vincula con el resto de las herramientas de intervención.

Al respecto, el ordenamiento territorial propone una necesaria rehabilitación y nueva conceptualización del Estado y de su rol imprescindible en los procesos sociales, ambientales y económicos. Un informe del Banco Mundial (1997: 7) lo pone en las siguientes palabras: "han fracasado los intentos de desarrollo basados en el protagonismo del Estado. Pero también fracasarán los que se quieran realizar a sus espaldas. Sin un Estado eficaz, el desarrollo es imposible". Se reivindica un nuevo tipo de Estado que guíe los procesos de desarrollo en un marco de ordenamiento territorial que garantice el consenso y participación de todos los actores involucrados. Se entiende la necesidad, así, de un Estado activo en donde se enfatice la intervención reguladora en este nuevo modo de gobernación (Majone, 1996: 1997).

Parece estarse alumbrando un consenso sólido reconocedor de que las imperfecciones o la ausencia de los mercados posibilita que la acción estatal mejore el rendimiento económico. Ahora bien, se trata de una simple posibilidad, pues una intervención estatal inadecuada podría empeorar dicho rendimiento. Uno de los círculos viciosos del no desarrollo procede de la convivencia de mercados imperfectos con procesos políticos todavía más imperfectos. Ante los fallos, la inexistencia o la insuficiencia de los mercados el problema no es, pues, si hay que intervenir o no, sino cómo asegurar la calidad de la intervención estatal (Stiglitz, 1994, citado en Ballabio, 2004: 70). 
Tabla 1

COMPARACIÓN ENTRE LOS ENFOQUES DE ORDENAMIENTO TERRITORIAL Y ECONOMÍA DEL TRANSPORTE

\begin{tabular}{|c|c|c|}
\hline & $\begin{array}{l}\text { Ordenamiento } \\
\text { Territorial }\end{array}$ & $\begin{array}{l}\text { Economía del } \\
\text { Transporte }\end{array}$ \\
\hline Definición & $\begin{array}{l}\text { Política pública y técnica } \\
\text { administrativa que busca ordenar } \\
\text { las actividades humanas en un } \\
\text { espacio geográfico capaz de } \\
\text { acogerlas }\end{array}$ & $\begin{array}{l}\text { Disciplina derivada de la teoría } \\
\text { microeconómica que analiza } \\
\text { y explica el comportamiento } \\
\text { y decisiones individuales en } \\
\text { relación con el transporte }\end{array}$ \\
\hline Objeto de estudio & Territorio & Mercado de transporte \\
\hline Modo de intervención & $\begin{array}{l}\text { Política de Estado transversal e } \\
\text { interadora de políticas sectoriales }\end{array}$ & Política sectorial de transporte \\
\hline Concepción del territorio & $\begin{array}{l}\text { Integral, multiescalar, } \\
\text { multidimensional y sistemática }\end{array}$ & $\begin{array}{l}\text { Omite naturaleza sistémica del } \\
\text { territorio. Considera únicamente } \\
\text { el espacio físico }\end{array}$ \\
\hline Concepción del Estado & $\begin{array}{l}\text { Activo, concertador de intereses } \\
\text { privados y públicos, busca mejorar } \\
\text { la calidad de vida }\end{array}$ & $\begin{array}{l}\text { Economicista, su objetivo es la } \\
\text { eficiencia en la asignación de } \\
\text { recursos, la eficiencia productiva y } \\
\text { preocupaciones distribuidas }\end{array}$ \\
\hline Subsistemas que potencia & Económico, social y ambiental & Económico \\
\hline Transporte colectivo & Subsistema del sistema territorial & Análisis de oferta y demanda \\
\hline Recursos productivos & $\begin{array}{l}\text { Infraestructura vial, } \\
\text { equipamiento móvil, recursos } \\
\text { humanos, energías, espacio } \\
\text { urbano y bienes ambientales }\end{array}$ & $\begin{array}{l}\text { Infraestructura vial, equipamiento } \\
\text { móvil, recursos humanos, } \\
\text { energías, espacio urbano } \\
\text { y bienes ambientales }\end{array}$ \\
\hline Actores que intervienen & $\begin{array}{l}\text { Demandantes reales y } \\
\text { potenciales, oferentes, Estado, } \\
\text { sociedad en general }\end{array}$ & $\begin{array}{l}\text { Demandantes reales, oferentes } \\
\text { y Estado }\end{array}$ \\
\hline Objetivos de intervención & Mejora en la calidad de vida & $\begin{array}{l}\text { Optimización del sistema } \\
\text { de movilidad a través de la } \\
\text { optimización del mercado de cada } \\
\text { modo de transporte }\end{array}$ \\
\hline
\end{tabular}

Fuente: Elaboración propia.

Desde el enfoque de la teoría microeconómica, asumido también por la economía del transporte, se adhiere a esta visión, aunque desde una perspectiva más enfocada en lo sectorial. 
Pero la perspectiva del ordenamiento territorial se busca el desarrollo conjunto de los subsistemas económico, social y ambiental. En donde estos subsistemas se integran se produce el desarrollo sostenible y se potencian las externalidades positivas y mitigan las externalidades negativas. Para alcanzar una integración entre dichos subsistemas en necesaria una coordinación entre los principios reguladores de cada uno de ellos. Cada subsistema tiene objetivos propios: equidad social; beneficios, eficiencia y máximo crecimiento económico y protección del medio ambiente. Al interrelacionarlos se pretende alcanzar: eficiencia asignativa (internalizando costos, protegiendo recursos estratégicos y valorando tanto las ventajas actuales como futuras), eficiencia distributiva (posibilitando a toda la población el acceso a los servicios urbanos para aprovechar las oportunidades de la aglomeración) y equidad ambiental (protegiendo los recursos físico naturales y permitiendo el acceso y disfrute de los mismos a toda la población tanto en sentido inter como intrageneracional). El ordenamiento territorial provee las bases legales, administrativas e institucionales para desarrollar en un territorio el modelo de movilidad elegido.

La teoría microeconómica es la que regula y enmarca los conceptos y fenómenos que ocurren en el subsistema económico, debiendo complementarse para hacer posible el ordenamiento territorial, con los postulados y principios de otras disciplinas que orientan a los otros subsistemas. De nuevo, la naturaleza multidisciplinar e interdisciplinar del ordenamiento del territorio tiene como resultado que este enfoque haga uso y adopte los conceptos y principios de la economía del transporte, enriquecidos, complementados y relacionados con aquellos de las otras disciplinas que entran en juego.

En esta línea de pensamiento, los sistemas de transporte tienen entre sus objetivos posibilitar a toda la población el acceso a los equipamientos urbanos y la realización de las actividades constitutivas de la vida. El transporte es, entonces, un promotor de la integración entre los subsistemas social y económico y su adecuado funcionamiento promueve el desarrollo sostenible de los territorios. Ligado a esto el ordenamiento territorial se orienta a asegurar la distribución equilibrada de los habitantes, las infraestructuras, equipamientos y actividades económicas, de esta manera sienta las bases para que los sistemas de transporte sean sostenibles. 
Las elecciones, en la economía del transporte, se realizan en condiciones de escasez. El ordenamiento territorial respecto del transporte considera como recursos escasos que intervienen en su mercado no solamente a aquellos tradicionalmente empleados (infraestructura vial, equipamiento móvil, recursos humanos, etcétera), sino también a la energía, el espacio urbano y los bienes ambientales. Un sistema de transporte sustentable satisface las necesidades de movilidad dentro de los límites de los recursos económicos, sociales y ambientales disponibles. En este punto, la economía del transporte coincide en la necesidad de una óptima asignación de los recursos escasos para desarrollar un modelo de movilidad eficiente. Si bien la economía del transporte no contemplaba a la energía, el espacio urbano y los bienes ambientales como recursos de producción, a lo largo del tiempo ha comenzado a incluirlos en sus análisis.

Uno de los aspectos innovadores del ordenamiento territorial es el abordaje del sistema de movilidad en conjunto, dejando de analizar a los modos de transporte por separado (como se realizaba en la planificación de transporte tradicional) y considerando los vínculos entre sus componentes. Esto permite que en el estudio de los usuarios de cada modo, ya no se consideren solamente a aquellos que efectivamente se trasladan en ese modo, sino que también se tomen en cuenta aquellos que potencialmente podrían ser usuarios. Tradicionalmente, en la economía del transporte se analizaba con mayor detenimiento a la oferta de servicios de transporte. El ordenamiento territorial propone hacer foco en la demanda, y no sólo real sino también potencial de movilidad. En este sentido, la economía del transporte aporta los conceptos, metodologías e instrumentos adecuados tanto para el análisis de la oferta (del servicio y de la infraestructura) como de la demanda (usuarios reales y potenciales). Si bien se encuentran más desarrollados los procedimientos para el estudio del transporte en su concepción tradicional y aún en su estadio conceptual los avances para el abordaje de la demanda potencial.

El mercado de transporte colectivo resulta de especial interés en tanto que es un servicio que traslada a gran cantidad de personas. Desde la disciplina económica, este mercado está integrado básicamente por tres agentes económicos. El primero se representa por la empresa prestadora de servicios de transporte de pasajeros u operador de servicios de transporte. Tales empresas tienen como finalidad el traslado de 
personas en un ámbito geográfico (urbano) a través de un medio físico (terrestre) y utilizando un modo de transporte determinado (autotransporte, ferrocarril, etc.). El segundo agente económico está compuesto por los consumidores, los cuales se representan por los usuarios de los distintos servicios ofrecidos por los operadores de transporte. Finalmente, el mercado está integrado por un tercer agente económico, el Estado, el cual interviene de manera directa e indirecta en la toma de decisiones tanto de los usuarios como de los operadores de los servicios de transporte (Islas Rivera, Rivera Trujillo y Torres Vargas, 2002). La economía del transporte reconoce al transporte colectivo como un servicio público y por tanto reconoce la necesidad de que su mercado esté mediado o regulado por el Estado para garantizar la prestación del mismo bajo determinadas condiciones. En palabras de Bergara (1999:7):

Los objetivos asociados a la actividad regulatoria en las industrias de servicios públicos pueden tener varias dimensiones: que se especifiquen estándares de calidad y seguridad en la provisión de los mismos, que se produzcan a bajo costo y que ese hecho sea trasladado a los consumidores en forma razonable, que se den procesos de inversión adecuados, etc. En una perspectiva de análisis económico, los objetivos relevantes para un regulador pueden sintetizarse en tres: eficiencia en la asignación de recursos, eficiencia productiva y preocupaciones distributivas.

El ordenamiento territorial reconoce la existencia de un mercado de transporte colectivo y la presencia de estos tres actores. Esta perspectiva considera al Estado como actor fundamental en este mercado ya que no confía en el libre juego de la oferta y la demanda, que, desde la perspectiva económica, está demostrado deriva en una competencia destructiva, con niveles de prestación bajos y en condiciones tecnológicas y ambientales precarias, no satisfaciendo a toda la demanda de transporte. De esta manera, al proponer un ordenamiento de la movilidad y del transporte colectivo dirigido por una figura estatal, reconoce la existencia de dicho mercado y la inadecuada asignación de recursos que a través del mismo se genera. Por estas razones, resulta de gran relevancia para el ordenamiento territorial conocer en detalle las características y el comportamiento de la demanda (real y potencial) y de la oferta de transporte, siendo la economía del transporte el enfoque por 
excelencia para llegar a este conocimiento. Es decir, el ordenamiento territorial hace uso de las herramientas y conceptos de la disciplina económica para entender el comportamiento de los mercados de transporte colectivo y plantea desde la misma los medios de intervención.

De esta manera, se detecta que los objetivos de intervención de ambos enfoques resultan coincidentes, por lo que los instrumentos de regulación utilizados son los mismos, si bien el ordenamiento territorial hace uso, asimismo, de instrumentos no económicos. Esto último implica, que este enfoque tiene entre sus objetivos la eficiencia en el mercado de todos los modos de transportes pero también tiene otros objetivos de intervención, entre los que se destacan fomentar el uso de modos sostenibles y planificar el modelo de movilidad en conjunto con los usos del suelo. Para alcanzar estos objetivos, pueden utilizarse, entre otros, (des)incentivos económicos planteados desde la economía del transporte.

\section{ECONOMÍA DEL TRANSPORTE EN EL ORDENAMIENTO TERRITORIAL}

Adicionalmente al análisis comparativo realizado, se detectan algunas particularidades del mercado de transporte que resultan interesantes mencionar, en tanto se vinculan con el territorio.

Por un lado, se observa que la demanda es derivada; es decir, en general (salvo en los viajes por ocio o placer) el traslado o desplazamiento no se demanda como un fin en sí mismo sino para realizar otras actividades. Estas actividades se localizan en diversos puntos del espacio geográfico y refieren al origen del vínculo entre transporte y territorio; lo que implica que la economía del transporte reconoce explícitamente la relación indisoluble entre transporte y usos del suelo.

Asimismo, esta demanda puede estudiarse en dos escalas: a nivel de mercado (representa a todos los usuarios del servicio) y a nivel individual (representa sólo a un usuario de transporte colectivo) (De Rus, Campos y Nombella, 2003). Interesa en este caso, estudiar la demanda de mercado que viene determinada por factores vinculados profundamente con el ordenamiento territorial: población, nivel de desarrollo económico, condiciones geográficas, usos del suelo, historia y cultura de dicha sociedad y política de transporte, o lo que es lo mismo, grado y modo de intervención del Estado en la regulación de la actividad. 
La población se vincula a sus características demográficas como su tamaño, tasa de crecimiento, distribución por grupos de edad, tipos de empleos, educación, etcétera y el nivel de desarrollo económico al nivel de actividad económica, concentración y ubicación de la misma, importancia relativa de cada sector. Mayor población y mayor nivel de actividad económica, en general, requieren de un grado mayor de movilidad y de transporte. El ordenamiento del territorio juega un papel fundamental en la determinación de estos factores. Respecto de la población genera medidas y acciones que la modifican con el objetivo de equilibrar la distribución de la misma en el territorio y de disminuir las brechas de ingresos. En particular, en las áreas urbanas busca su consolidación y crecimiento planificado y regulado, mitigando los efectos negativos del crecimiento espontáneo y descontrolado. Estas intervenciones resultan favorables para el sistema de transporte colectivo en tanto que la demanda se encuentra contenida en un espacio geográfico delimitado. Respecto del desarrollo económico, fomenta las actividades en las que los territorios tienen vocación y ventaja comparativa, buscando promover y localizar las mismas considerando la capacidad de acogida del territorio. Esto repercute positivamente en el nivel de actividad económica que tiene como consecuencia la mayor demanda de traslados en el territorio.

Las condiciones geográficas particulares de un territorio pueden facilitar o dificultar determinadas infraestructuras que condicionan los modos de transporte que se implementarán. A través del ordenamiento territorial no sólo se conocen dichas condiciones geográficas sino que se establece, en función de ellas y del modelo urbano deseado, a diferentes escalas geográficas, la localización y el tipo de las infraestructuras de transporte óptimas para un territorio. De esta manera se prevé que la movilidad y el servicio de transporte de un territorio urbano no sea interrumpida por factores físico naturales (inundaciones, lluvias, nevadas, sismos, etcétera). Se facilita y garantiza, así, el acceso a la red de movilidad, lo cual favorece a la demanda de transporte.

Por su parte, los usos del suelo tendientes a la homogeneidad de los territorios tienen una demanda de movilidad mayor a aquellos territorios heterogéneos en sus usos, actividades y funciones, en donde todo se localiza en proximidad. A su vez, la dispersión de los usos del suelo en el territorio requiere de mayor nivel de movilidad que la compacidad de los mismos. El ordenamiento territorial urbano tiene el objetivo de 
reducir los requerimientos de movilidad a través de la consolidación de los espacios urbanos, fomentando, entonces, los usos del suelo mixtos, cuando esto es posible. Una ciudad compacta y ordenada resulta sostenible y favorece el desarrollo eficiente y equitativo de los modos de transporte colectivo y no contaminantes. La demanda de transporte de un territorio con estas características se encuentra equitativamente distribuida en el mismo facilitando la operación de estos modos. Es decir, la distribución de los usos del suelo de acuerdo a los criterios del ordenamiento territorial fomentan la demanda de transporte más sostenible.

La historia y cultura de una sociedad dan cuenta de los usos y costumbres respecto de los modos de movilidad utilizados y la consciencia social y ambiental de la misma. Una sociedad con interés por el conjunto de la comunidad tiende a utilizar modos más sostenibles que una sociedad que persigue solamente los intereses individuales. En este sentido, el ordenamiento territorial aboga por una mayor consciencia social y ambiental que promueve el uso prioritario de los modos colectivos dentro del sistema de movilidad.

Respecto de la política de movilidad y transporte, considerada como la regulación de los mercados de cada modo de transporte, establece las condiciones bajo las cuales pueden prestarse legalmente los servicios de transporte o explotarse las infraestructuras, condicionando las decisiones de los demandantes y oferentes. En un contexto de ordenamiento territorial todas estas acciones se orientan hacia el modelo de movilidad sostenible: promoción de modos colectivos y no contaminantes y desincentivo al uso de modos particulares.

Otra característica distintiva respecto de otros mercados es la consideración del tiempo. El tiempo invertido por los usuarios del sistema de transporte influye en la valoración y elección que éstos hacen del modo de transporte (el tiempo no es fijo en tanto que el usuario puede elegir entre diversas alternativas con distintos tiempos para un mismo trayecto). Desde la perspectiva del ordenamiento territorial el tiempo resulta un recurso fundamental que debe ser empleado racionalmente, por lo que se busca la reducción de los desplazamientos a través de la mezcla de usos y funciones para que todas las actividades se desarrollen en proximidad. Esto permite que a lo largo del día se destine menos tiempo al traslado y más tiempo a las actividades, entendiendo que éstas favorecen el bienestar de las personas. Es decir, el objetivo 
que tiene el ordenamiento territorial se complementa con la necesidad de reducir los tiempos de viaje que supone la economía del transporte.

Finalmente, por el lado de la oferta, desde la economía del transporte se han comenzado a considerar recursos escasos que tradicionalmente no se tenían en cuenta y que permiten un vínculo más estrecho con el ordenamiento territorial. Entre ellos están los recursos naturales (ambiente) y el suelo urbano ocupado. Éstos son considerados claves para que el sistema de transporte sea eficiente y permiten internalizar externalidades positivas y negativas.

\section{APLICACIÓN DE CONCEPTOS Y METOdOLOGÍA DE LA ECONOMÍA DEL TRANSPORTE}

En la consideración de la relación sistémica de la oferta y la demanda de transporte, se observa que la elección del modo de transporte depende, entre otras cosas, de quién asume realmente el costo de producción del viaje. Dada la existencia de externalidades negativas, sobre todo en los modos motorizados privados, si éstas no se incorporan al mercado y las asume quien las genera, se producen incentivos para el mayor uso de estos trasportes en perjuicio de los modos colectivos, considerados más equitativos y sostenibles. Por ello, una política pública que integre a todos los componentes del sistema de movilidad, y en el marco de un ordenamiento territorial, podría guiar los incentivos para que los usuarios prefieran unos modos sobre otros. De esta manera, se podría conseguir que el costo de producción de un viaje en automóvil particular internalizara los costos marginales que se imponen al resto de la sociedad (congestión, mayor uso del espacio público, mayor generación de emisiones contaminates) y beneficiara así la elección de los modos colectivos.

En las ciudades de América Latina (tabla 2) se detecta, en general, que el costo de producción por viaje en automóvil es más alto que en transporte colectivo, generando un incentivo al uso de este último. Sin embargo, en muchos casos los costos de ambos modos son similares. En las ciudades de Europa (tabla 3) la diferencia de costos es significativamente mayor, favoreciendo de manera más contundente el uso de modos colectivos.

Por otro lado, desde la perspectiva de la economía del transporte se puede detectar la existencia de economías de escala en la prestación del 
servicio colectivo, que da lineamientos para definir una política pública que favorezca su eficiente funcionamiento. Se constata en la literatura (de Grange, Troncoso y Briones, 2018) que no existe un patrón de comportamiento generalizado, sino que la presencia de economías de escala depende de las características del mercado de transporte, como así también de las particularidades del territorio y la disponibilidad de información. Se presenta el caso concreto del área metropolitana de Mendoza, ubicada en la provincia de Mendoza, Argentina, en cuyo territorio el transporte colectivo opera con deseconomías de escala, lo que implica que el servicio funcionaría de manera más eficiente si lo prestaran una mayor cantidad de empresas (García Schilardi, 2012). Se observa, entonces, una tendencia hacia la concentración empresaria a lo largo del tiempo, por lo que se requiere de adecuada intervención estatal para que se alcance una situación de mayor eficiencia y también de equidad. Esto sería posible a través de la integración de la movilidad y el transporte en la política de ordenamiento territorial, con marco legal vigente en este territorio desde el año 2009 (Ley de Ordenamiento Territorial y Usos del Suelo $\left.N^{\circ} 8051 / 09\right)$. Las deseconomías de escala se detectan estimando la función de costos a partir de una función de tipo translog, perteneciendo esta metodología típicamente a la economía del transporte.

Finalmente, es oportuno mencionar el fuerte impacto que tiene el avance de la tecnología en el mercado de transporte, beneficiando tanto a la demanda como a la oferta. La primera en términos de la disponibilidad de información para usuarios respecto de recorridos, horarios, frecuencias y abono de boletos. De esta manera la demanda puede hacer uso más eficiente del servicio a través de aplicaciones móviles disponibles y asequibles para todos los usuarios. La oferta se beneficia a través de la demanda que resulta más flexible en tiempo y espacio, a la vez que también resulta más adaptable a ésta por el uso de vehículos con nuevas características técnicas de tamaño, menor contaminación y adecuación a todo tipo de usuarios y mayores posibilidades de funcionamiento en red. Resulta fundamental, entonces, que la economía del transporte actualice sus conceptos para incorporar estos nuevos componentes de tan alto impacto. 
Tabla 2

Costos POR VIAJE SEgún MOdOS DE TRANSPORTE EN CIUDADES DE AMÉRICA LATINA

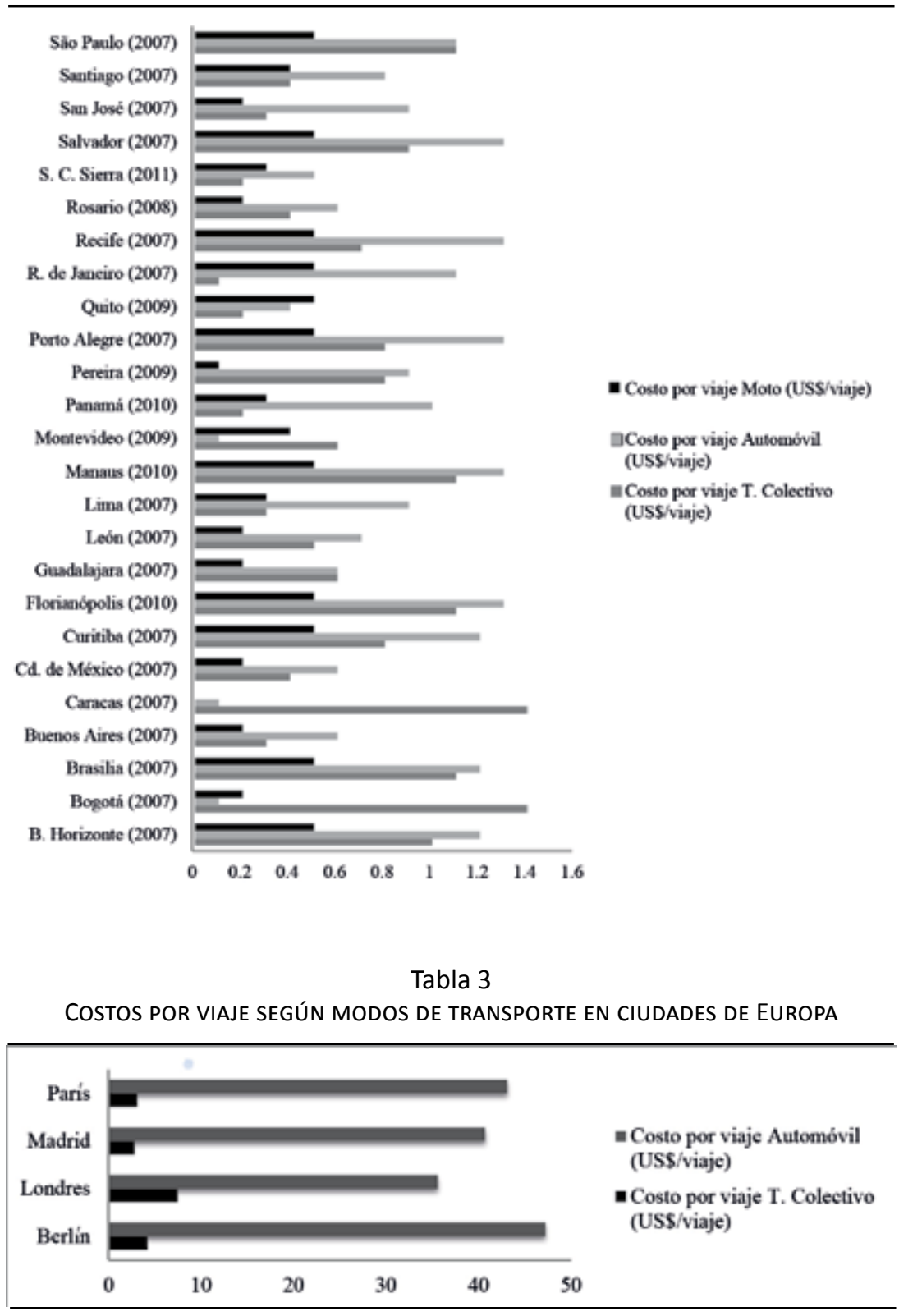

Fuente: Estupiñán et al., 2018. 


\section{REFLEXIONES FINALES}

El ordenamiento territorial resulta una alternativa efectiva frente a las intervenciones sectoriales, voluntaristas y centralizadas en el Estado que han impedido el desarrollo. Se plantea como una política que perdura en el tiempo y que trasciende las ideologías de los gobiernos que la llevan adelante. Propone un nuevo tipo de Estado que guíe los procesos de desarrollo en un marco de consenso y participación de todos los actores involucrados. Busca armonizar la acción del sector público con el interés de los agentes privados, el interés privado con el interés colectivo y conciliar los objetivos puramente económicos con los de las dimensiones social y ambiental. Para esto se requieren políticas públicas, previstas en el ordenamiento territorial, que entiendan la naturaleza multiescalar, sistémica e integral del territorio y que racionalicen el proceso de decisiones de corto, mediano y largo plazo, lo que exige una coordinación y coherencia en el accionar de las diversas instituciones y actores involucrados. De esta manera, se asegura la calidad de la intervención estatal, superando la histórica debilidad que ha tenido el Estado frente al mercado y que ha generado en todas las escalas, en particular en la urbana, grandes desequilibrios territoriales, inequidades sociales y deterioro ambiental.

Asimismo, se advierte que tanto la movilidad como el transporte han sufrido cambios en su dimensión analítica como aplicativa enmarcados en el nuevo paradigma de la sostenibilidad (Miralles Guasch, 2013). En este contexto la economía del transporte asume una visión de transporte sostenible afirmando la influencia del mismo en la economía, en la sociedad y en el ambiente. Lo considera un servicio público que responde al principio de equidad resultando de gran importancia e impacto en las dimensiones económica, social y ambiental. En estas consideraciones se plantea la necesidad de un transporte en red, considerando todos los modos en conjunto, que se traduce en economías de costos (por ejemplo cuando crece el número de usuarios del transporte colectivo que facilita mayores frecuencias que reducen los tiempos de espera) y en la obtención de economías de escala (referidas también al transporte colectivo y asociadas al diseño de líneas troncalizadas con sistema de alimentación). Asimismo, se estima indispensable la regulación del transporte para garantizar la adhesión de éste al principio de equidad. Se evidencia, entonces, que la economía del transporte es una 
disciplina necesaria para el ordenamiento territorial, que provee herramientas conceptuales, metodológicas y teóricas para el entendimiento del mercado de los diversos modos de transporte.

Se entiende que el ordenamiento territorial requiere de un abordaje interdisciplinario, holístico e integral. Por lo tanto, y por lo que se ha argumentado, se detecta que la economía del transporte resulta un enfoque adecuado y útil a los fines del abordaje de la dimensión económica aunque requiere ser complementado con otros instrumentos para poder dar una respuesta adecuada a los problemas de la movilidad. Es decir, la perspectiva del ordenamiento territorial comprende al análisis económico y a sus instrumentos, pero es un enfoque más amplio que se vale, además, de otras herramientas y conceptos para sus estudios y políticas.

\section{Bibliografía}

Almeida García, Fernando (1999). Análisis y propuestas de ordenación territorial para la costa del sol oriental: el conflicto entre la agricultura y el turismo. Tesis doctoral. Departamento de Geografía, Universidad de Málaga. España.

Ballabio, Marcela (2004). Los determinantes de selección individual de modo de transporte en la ciudad de Mendoza. Tesis de maestría. FLACSO, México.

Banco Mundial (1997). El Estado en un mundo en transformación. Washington D.C.: Banco Mundial.

Bergara, Mario (1999). La regulación de los servicios públicos. Una nota introductoria. Universidad de la República, Departamento de Economía, Facultad de Ciencias Sociales. Montevideo.

Consejo de Europa, Conferencia Europea sobre Ciudades Sostenibles (1983). Carta Europea de Ordenación del Territorio. Recuperado de: http://www. ehu.eus/Jmoreno/ArchivosPOT/CartaEuropeaOT.pdf

De Grange, L; R. Troncoso e I. Briones (2018). "Cost, production and efficiency in local bus industry: An empirical analysis for the bus system of Santiago," Transportation Research Part A: Policy and Practice, 108, 1-11.

De Rus, Gines; Javier Campos y Gustavo Nombella (2003). Economía del transporte. Barcelona, España: Antoni Bosch.

Estupiñán, N.; H. Scorcia; C. Navas; C. Zegras; D. Rodríguez; E.Vergel-Tobar; R. Gakenheimer; Z. Azán Otero y E. Vasconcellos (2018). Transporte y Desarrollo en América Latina. 1 (1). CAF Banco de Desarrollo de América Latina. 
García Schilardi, M. E. (2012). Costos del transporte colectivo y rendimientos a escala. Caso: área metropolitana de Mendoza. Tesis de maestría en economía urbana. Universidad Torcuato Di Tella, Buenos Aires, Argentina.

Gómez Orea, Domingo; Alejandro Gómez Villarino y María Teresa Gómez Villarino (2014). Marco conceptual de la Ordenación del Territorio. II Curso Internacional de Postgrado de Ordenamiento Territorial. Fundabaires.

Gómez Orea, Domingo (2002). Ordenación Territorial. Madrid: Mundi-Prensa y Agrícola Española S.A.

Gudiño, María Elina (2015). “El Ordenamiento Territorial como política de Estado”. Perspectiva Geográfica, 20 (1), pp. 11-36.

Gudiño, María Elina (2010). "Del urbanismo reglamentario a las nuevas concepciones del ordenamiento territorial”. Scripta Nova. 14 (331).

Gudiño, María Elina (2009). "Instrumentos para la gestión del territorio. Ley de ordenamiento territorial y sistemas de información geográfica”. Jornadas Regionales de Información Geográfica y Ordenamiento Territorial, Ministerio Secretaría General de la Gobernación, Proyecto SIT Santa Cruz.

Gudiño, María Elina (2002). “Comentarios y Conclusiones del I Seminario Internacional. La Interdisciplina en el Ordenamiento Territorial ”, Proyección. 1 (2).

Gutiérrez, Andrea (2009). “Movilidad o inmovilidad: ¿qué es la movilidad? Aprendiendo a delimitar los deseos”, XV CLAPTU. Buenos Aires.

Herce, Manuel (2009). Sobre la movilidad en la ciudad. Propuestas para recuperar un derecho ciudadano. Barcelona: Editorial Reverté.

Islas Rivera, Víctor; César Rivera Trujillo y Guillermo Torres Vargas (2002). Estudio de la demanda de transporte. Sanfandila, México: Secretaría de Comunicaciones y Transportes. Instituto Mexicano del Transporte (Nro. 213).

Ley $\mathrm{N}^{\circ} 8051$. Boletín oficial de la provincia de Mendoza, Mendoza, Argentina, 22 de mayo de 2009.

Majone, Giandomenico (1996). Regulating Europe. London: Routledge.

Majone, Giandomenico (1997). "From the Positive to the Regulatory State: Causes and Consequences of change in the mode of governance", Journal of Public Policy, 17. pp. 139-167.

Massiris Cabeza, Ángel (2005). Fundamentos conceptuales y metodológicos del ordenamiento territorial. Colombia: Universidad Pedagógica y Tecnológica de Colombia.

Massiris Cabeza, Ángel (1997). “Ordenamiento Territorial, Región y Procesos de Construcción Regional”, Revista Perspectiva Geográfica, No. 1, pp. 7-87.

Miralles Guasch, Carmen (2013). "Presentación: Dossier Metodologías y Nuevos Retos en el Análisis de la Movilidad y el Transporte", Transporte y Territorio. 8, pp. 1 - 6. 
Miralles Guasch, Carmen (2002). Transporte y Ciudad. El binomio imperfecto. Barcelona: Ariel.

Montezuma, Ricardo (1999). Bogotá: ciudad y movilidad. El papel del transporte en el proceso de urbanización, 1884-1998. Bogotá: CEJA-INJAVIU.

Raffestin, Claude (1980). Pour une Geographie du Pouvoir. Francia: Librairies Techniques.

Romero, Hugo y Alexis Vázquez (2005). "Pertinencia y significado del ordenamiento territorial en Chile", Urbano. 8 (011).

Salado García, María Jesús; María de los Ángeles Díaz Muñoz; Joaquín Bosque Sendra; Carolina Carvalho Cantergiani; C. Rojas Quezada; F. J. Jimenez Gigante; I. Barnetti; C. Fernández; C. Muñoz Rueda (2002). Movilidad Sostenible y SIG. Propuesta de Evaluación del Transporte Público en Alcalá de Henares. Madrid: Departamento de Geografía. Universidad de Alcalá de Henares.

Santos, Milton (2000). La naturaleza del Espacio. España: Editorial Ariel.

Santos, Milton (1996) La naturaleza del espacio. Técnica y tiempo. Razón y emoción. Ariel, Barcelona. 